\title{
Modeling of a UAV-based Data Collection System
}

Antonia Arvanitaki and Nikolaos Pappas

The self-archived postprint version of this conference article is available at Linköping University Institutional Repository (DiVA):

http:/ / urn.kb.se/ resolve?urn=urn:nbn:se:liu:diva-147967

N.B.: When citing this work, cite the original publication.

Arvanitaki, A., Pappas, N., (2017), Modeling of a UAV-based Data Collection System, 2017 IEEE 22ND INTERNATIONAL WORKSHOP ON COMPUTER AIDED MODELING AND DESIGN OF COMMUNICATION LINKS AND NETWORKS (CAMAD).

https:// doi.org/ 10.1109/CAMAD.2017.8031526

Original publication available at:

https:/ / doi.org/ 10.1109/ CAMAD.2017.8031526

Copyright: IEEE

http:// www.ieee.org/

(C) 2017 IEEE. Personal use of this material is permitted. However, permission to reprint/ republish this material for advertising or promotional purposes or for creating new collective works for resale or redistribution to servers or lists, or to reuse any copyrighted component of this work in other works must be obtained from the IEEE. 


\title{
Modeling of a UAV-based Data Collection System
}

\author{
Antonia Arvanitaki* and Nikolaos Pappas ${ }^{\dagger}$ \\ *Department of Computer and Information Science, Linköping University, Linköping, Sweden \\ ${ }^{\dagger}$ Department of Science and Technology, Linköping University, Norrköping, Sweden \\ \{antonia.arvanitaki, nikolaos.pappas\}@liu.se
}

\begin{abstract}
Unmanned Aerial Vehicles (UAVs) have been extensively studied the past years for various applications. In this work, we propose a Markov chain to model the movement of a single UAV deployed for data collection from remote sensors. Furthermore, we introduce a second Markov chain to model the irregularities of the UAV's movement when it is in transit. We investigate the impact of the distance of the UAV from the sensor on the success probability of information transmission. We provide numerical evaluation of the theoretical results.

Index Terms-UAV, Discrete Time Markov Chain, Sensor network, Mobility Modeling, Data Collection.
\end{abstract}

\section{INTRODUCTION}

Since their emergence, Unmanned Aerial Vehicles (UAVs) have been deployed in military, commercial and public applications. Particularly, they have been used in military missions replacing pilots in order to reduce losses. They can also be employed to facilitate real-time end-to-end communication in the absence of infrastructure in cases of disasters or in extreme environments. Other applications in the commercial domain include monitoring and surveillance of remote areas. In the public domain, UAVs can assist in the public safety.

\section{A. Background and Related Work}

Therefore, many challenges arise in the wireless communication with UAVs. Gupta et al. [1] study the significant questions that arise in UAV communication networks about the features of the UAV networks, the routing, the handover schemes and the energy efficiency in UAV networks. Zeng et al. [2] discuss open issues for further research regarding the UAVs. They present the basic networking architecture, the main design considerations like UAV deployment, path planning, and communication schemes with controlled mobility.

Existing work approaches the aspect of designing mobility models for the UAVs in order to improve the end-to-end communication. Fadlullah et al. [3] address the problem of using UAVs as communication network facilitator in disaster scenarios, where no access to cellular and internet infrastructure are available. The authors propose a dynamic trajectory control algorithm for the UAVs, which achieves a higher probability of end-to-end communication. Instead, Yanmaz [4] assumes that the UAVs control their own trajectory and a probabilistic mobility model is introduced for the UAVs. A decentralized mobility control algorithm [5] is also introduced in order to use the UAVs as communication relays which achieve an optimal end-to-end communication.

One of the aspects investigated is the communication scheme. Ho et al. [6] discuss about the problem of finding

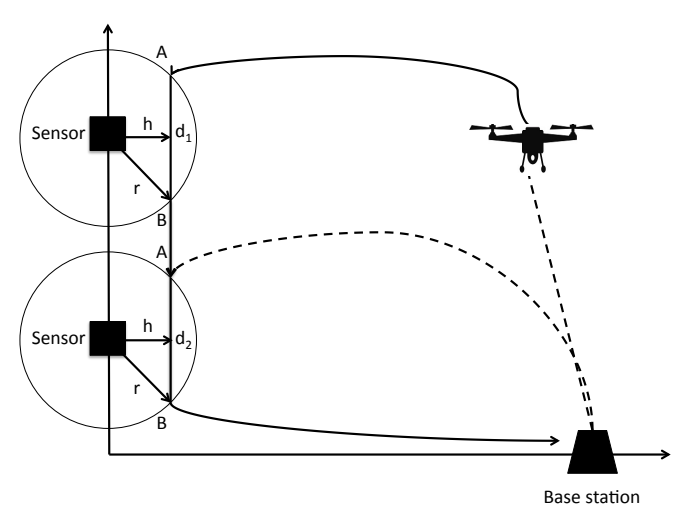

Fig. 1: The model of the network: an illustrative case.

the best relay UAVs in a cluster with UAVs. Li et al. [7] study a multi-hop device to device communication scheme using UAVs as relays for addressing the problem of coverage of areas where land relays cannot be deployed. Olsson et al. [8] address the problems of limited communication range and high interrupted bandwidth. They propose relay chains for the supervision of a sole target and relay trees for concurrent supervision of numerous targets, forwarding the information to the base station. Jawhar et al. [9] introduce different models and strategies for data collection in a cluster-based wireless sensor network with UAVs. Their goal is to reduce the energy consumption, the radio frequency interference, as well as achieve a longer network lifetime.

The communication performance of the UAVs constitutes a key factor to the reliability of the UAV networks. Horiuchi et al. [10] study the communication performance in terms of throughput and they formulate an optimal location of the relay UAV that maximizes the throughput. The network consists of a base station, a relay UAV and an observation UAV, and the data transmission is real-time. Ono et al. [11] investigate the scenario where a fixed-wing UAV connects two isolated base stations. It can complement the cellular and satellite networks, as well as participate in rescue missions for disaster scenarios. The authors address the problem of the performance limitation in terms of outage probability and data rate, when the UAV relays the data to the second base station. Giorgetti et al. [12] introduce an energy-efficient communication scheme for a wireless sensor network and a single UAV. The sensors send 
the same message to a Rake receiver located on the UAV, which fuses the weak and noisy signals. The scheme achieves energy efficiency and communication reliability.

Another issue addressed is the deployment of the UAVs. Mozaffari et al. [13] propose a deployment scheme for the UAVs, which have directional antennas and act as base stations. Their role is to provide coverage to ground users. The authors optimize the deployment of the UAVs, such that the coverage of the ground users is maximized. Chen et al. [14] study the scenario where UAVs are used in order to provide cellular or internet service to mobile users in CRAN system.

Energy-efficient networks have also been on focus of current research. Li et al. [15] study the problem of energy balancing in a network with UAVs, acting as communication relays in real-time applications. The goal of this paper is to extend the network lifetime. For this reason, the authors propose an energy-efficient cooperative relaying scheme and an energy efficient scheduling.

The scheduling of the UAVs in cooperative UAV networks has been extensively studied. Li et al. [16] propose an energyefficient cooperative relaying scheme using UAVs for data collection from a remote sensor network. The UAVs relay the data to a base station. The optimal scheduling problem is formulated, which is generated in the base station and has guaranteed success rates and balanced energy consumption. A scheduling analysis framework [17] is proposed to check the coverage of a network of ground-based nodes by a single UAV. If this fails, then a delay tolerant network (DTN) is formed in order the UAVs collect, buffer, and deliver the data.

Powel et al. [18] introduce the collaboration of UAVs and humans for emergency scenarios and introduce a multi-server queuing for surveillance. An extension of 802.11 networks [19] is suggested for enabling communications among UAVs, which move in $3 \mathrm{D}$ aerial space. Its performance is analyzed in terms of throughput and the number of retransmissions. Jeong et al. [20] propose a UAV-based mobile cloud computing system for uplink and downlink transmissions to stationary users located in areas with limited or no infrastructure. The authors formulate an optimization problem for the minimum mobile energy over the bit allocation for the offloading services and over the UAV's path for the two access schemes that are applied.

Markov chains have been previously introduced in studying the performance of the UAVs. Yanmaz et al. [21] study the sensor coverage performance in a UAV-assisted wireless sensor network with multiple UAVs. More precisely, the authors introduce a Markov chain in order to study the coverage performance of diverse mobility patterns for the UAV. The performance metrics they adopt are the coverage, average, and full distributions.

The UAVs might play an important role in smart metering applications [22]. A smart metering system can take electricity, water, and gas measurements [23]. It is responsible for reading and recording the consumption with timestamps, as well as reporting, monitoring and customer billing. The meters that are currently used (e.g. rugged handhelds), are energy-constrained and have a short range for signal transmission, which leads to an important delay of data transmission to the utility server. A significant question that arises is whether the rugged handhelds can be replaced by the UAVs, or they can cooperate in collecting data efficiently. Since the UAVs have a longer battery lifetime and a longer communication range, they are suitable for such applications. Therefore, it is important to describe thoroughly the performance and the movement of the UAVs.

\section{B. Contribution}

In this work we study the performance of a UAV-assisted sensor network. More specifically, we consider the scenario where sensors are spread in the environment in distant/isolated or difficult to reach areas. The sensors collect data and store it in their limited-capacity storage equipment. The sensors do not have connection with a base station or among them. Thus, a $\mathrm{UAV}$ is deployed to collect the data from the sensors and then to transfer it to the base station. The data collection takes place through wireless transmission when the UAV is in proximity to the sensor. We model the movement of the UAV as a discrete time Markov chain. Furthermore, during the transition of the UAV between the sensors and/or the base station we model the irregularities of the movement due to random events (weather, failures, etc.) as another Markov chain. Finally, we provide numerical evaluation of the theoretical analysis.

\section{SySTEM MODEL}

\section{A. Network Model}

We consider a network with $i$ sensors, where $i=1,2$. We also assume a single UAV and a single base station. The sensors use the UAV as a relay to transmit data to the base station. The model of the network is depicted in Fig. 1.

The sensors have limited energy, and communication capabilities. They are located at fixed positions so the distances are known. They are equipped with a detector that detects the UAV when it comes in proximity with the sensors and when it leaves their communication range. The sensors sense with a fixed rate and store the data in their buffer. Each sensor generates $\lambda_{i}$ packets/slot and it has a finite buffer capacity. The capacity of the buffer is $B_{i}$ and it takes $\frac{B_{i}}{\lambda_{i}}$ slots to get full. We assume that $\lambda_{1}=\lambda_{2}=\lambda$ and $B_{1}=B_{2}=B$. The sensors might be located in close distance from each other or long distance. In this work we consider the case of the long distance. This can capture the case that instead of sensors we have aggregators that are collecting data from sensors nearby them, then the aggregators are responsible to send the collected data to the UAV.

We assume that the sensors have limited capabilities and they cannot collect data while they transmit to the UAV. When the transmission of the data is completed or when the UAV leaves the proximity area, the sensor deletes the transmitted content and starts the measurements again.

The UAV is assumed that it starts its mission from the base station. Its role is to store the data that it receives from the sensors and transmit it to the base station, the data 
transmissions take place when the UAV and the sensors/base station are in proximity. In this work we assume that the UAV is in proximity with a sensor or the base station when the success probability of a packet transmission is above a given threshold. The reason for this is to reduce the energy consumption at the UAV, since the UAV can disable the communication module when is not used. The expression for the success probability is provided later. The UAV does not process the data, it just collects and delivers it. It has an infinite size buffer $B_{U}$. This is justifiable since the UAV has more capabilities than a sensor. The UAV travels with an average velocity $v$ from the base station towards the sensors and back to the base station. We assume that the UAV and the sensors have the same communication interface, so they can communicate. The UAV moves in the communication range of the sensor $i$ and it collects the stored data. Within a trip from the station and back, the UAV can visit a sensor at most one time.

During the data collection from a sensor, the UAV remains in proximity as long as it is required to collect the data. ${ }^{1}$ After the collection of the data, the UAV flies back to the base station to deliver the data and to perform regular maintenance tasks such as charging etc. In this study, we consider only the uplink transmission between the sensors and the UAV. A common assumption that we adopt is that the communication bandwidth between the UAV and the base station is high, so the collected data is transmitted succesfully very fast. We can also assume that when the UAV is in proximity with the base station, the channel between them is modeled as line-of-sight channel (LOS). The UAV and the base station can communicate using the communication technology such as IEEE 802.16 (WiMax), cellular, etc.

\section{B. Channel Model}

We assume that the channel between the sensors and the UAV is subject to path loss and Rayleigh fading. ${ }^{2}$ A sensor $i$ transmits successfully a packet to node $j$ (UAV) if and only if $\operatorname{SINR}(i, j) \geq \gamma_{j}$, where $\gamma_{j}$ is a threshold depending on characteristics such as the transmission rate, modulation and other physical layer characteristics. The transmit power of the sensor $i$ when it transmits to the $\mathrm{UAV}$ is $P_{i}^{U}$. The received power at the UAV when the sensor $i$ transmits is $P(i, j)=A(i, j) h(i, j)$, where $A(i, j)$ is a random variable that represents small-scale fading and is exponentially distributed under the Rayleigh fading. The received power factor is derived by $h(i, j)=P(i)(r(i, j))^{-\alpha}$, where $\alpha$ is the path loss exponent and $r(i, j)$ is the distance between the sensor $i$ and the node $j$ (UAV). The success probability is given by

$$
P_{i}^{j}=\exp \left(-\frac{\gamma_{j} \eta_{j}}{v(i, j) h(i, j)}\right)
$$

\footnotetext{
${ }^{1}$ In a future extension of this work we will allow also a percentage of packet dropping under certain circumstances.

${ }^{2}$ However, the analysis we provide in this work is independent of the details of the channel, so it can be used for other channels as well.
}

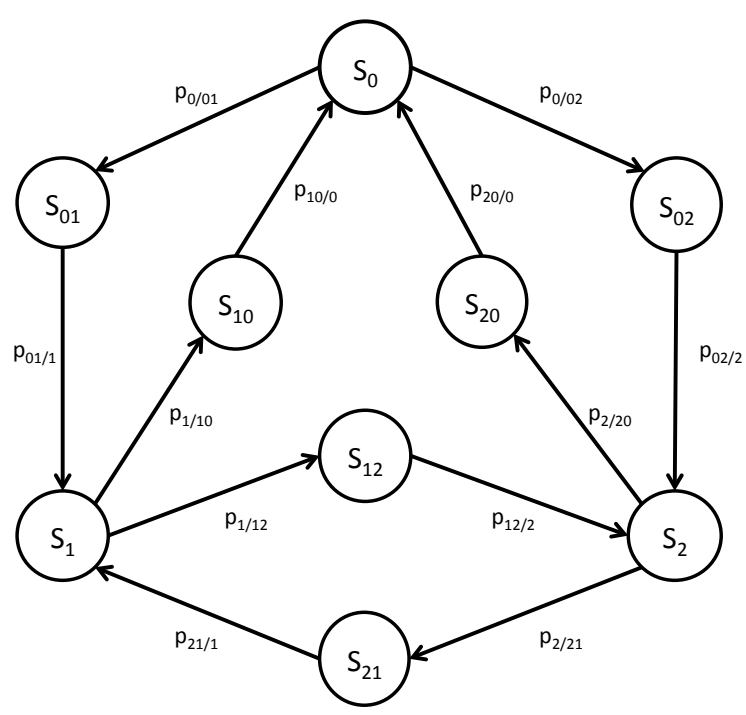

Fig. 2: The discrete time Markov chain that models the state of the UAV.

where $v(i, j)$ is the parameter of Rayleigh fading random variable, and $\eta_{j}$ is the receiver noise power at $j$.

\section{Performance Analysis}

In this section a discrete time Markov chain model is presented, which captures the status of the UAV. The UAV moves from the base station to the sensors in order to collect data and back to the station to transfer them and perform maintenance tasks.

\section{A. Markov Chain Model}

We introduce a discrete time Markov chain (DTMC) that models the mobility status of a UAV depicted in Fig. 2. The states $S_{i}$ where $i=0,1,2$ model the case where the UAV is either at the base station $\left(S_{0}\right)$ or collecting data from sensor $i\left(S_{i}\right)$. More specifically, when the UAV is at the state $S_{0}$, it means that it is at the base station recharging and/or transmitting the collected data from the sensors. We assume that the time for the UAV to deliver the data to the base station is shorter than the recharging time. ${ }^{3}$

Let us refer to a sensor or the base station as a node here. The state $S_{i j}$ denotes a transition from node $i$ to $j$. The transition probability from the state $i$ to the state $j$ is denoted by $p_{i / j}$. The state $S_{i}$, where $i=1,2$, models the case where the UAV is in the communication range of sensor $i$. The sensor $i$ sends the data to the $\mathrm{UAV}$, and when the transmission finishes, the UAV moves to the state $S_{i j}$.

The UAV flies along a path from node $i$ to node $j$. The route $i \rightarrow j$ is not necessarily the same as the route $j \rightarrow i$ due to potential obstacles, or weather conditions.

\footnotetext{
${ }^{3}$ It is not difficult to connect the charging time with the average sojourn time of $S_{0}$, but we disregard this part here.
} 


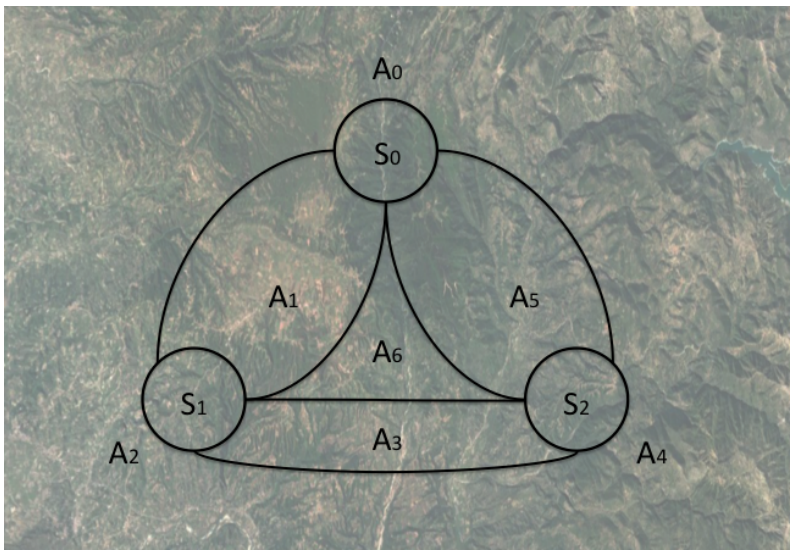

Fig. 3: Partitions of the possible locations of the UAV.

We divide the area with the deployed sensors and the base station into partitions as illustrated in Fig. 3. Let us denote $A_{i}$ the partition $i$. We consider that the partitions $A_{2}$ and $A_{4}$ correspond to the states $S_{1}$ and $S_{2}$ respectively, in which the UAV is in proximity with the sensor $i$, where $i=1,2$ respectively.

We introduce a discrete time Markov chain (DTMC), which models the movement of the UAV in the partitions $A_{1}, A_{3}$, and $A_{5}$ as shown in Fig. 4. This DTMC can be seen as another model that lies inside the states $S_{i j}$ in Fig. 2. The parameters in this Markov chain model the variations in the flying speeds due to air, weather, malfunctions of the hardware or cases of emergency. The state Away depicts the scenario where the UAV moves in a way that increases the distance from the destination node with probability $p_{d}$. When the UAV is in the state Current Position, it hovers above its location and the distance to the destination does not get shorter or longer, with a probability $p_{s}$. The UAV is in the state Closer, when it moves closer to the sensor $i$ or the base station with probability $p_{c}$.

The steady-state probability vector $\pi$ can be obtained by solving the set of linear equations. ${ }^{4}$ The first nine equations are given by

$$
\sum_{i} \pi_{i} P_{i j}=\pi_{j}
$$

where $i, j=0,01,10,1,2,02,20,12,21$. The tenth is given by

$$
\pi_{0}+\pi_{01}+\pi_{10}+\pi_{1}+\pi_{2}+\pi_{02}+\pi_{20}+\pi_{12}+\pi_{21}=1
$$

The mean time that the UAV needs to travel the distance $d_{i j}$ from the node $i$ to the node $j$ with constant velocity $v_{i j}$ is given by

$$
t_{i j}=\frac{d_{i j}}{v_{i j}}
$$

$$
\text { where } i=0 \text { and } j=1,2 \text { or } i, j=1,2 \text {. }
$$

\footnotetext{
${ }^{4}$ Due to the space limitations we omit the detailed derivation of the steady state probability vector.
}

The distance that the UAV will have from the sensor $i$ while communicating with it, depends on the success probability given in (1). More specifically, the probability that the UAV's decoder receives successfully a packet becomes higher when the distance $h$ between the sensor $i$ and the UAV is shorter. The path length that the UAV will have in coverage of the sensor $i$ is given by

$$
d_{i}=2 \sqrt{r^{2}+h^{2}}
$$

The average time that the UAV spends in the communication range of the sensor $i$ depends on the data that the sensor $i$ has in its buffer to be transmitted and the trajectory that the UAV follows. More specifically, the distance $d_{i}$ depends on the communication range of the sensor $i$ and the height $h$ as presented in (5).

The total average time that the UAV spends flying between two consecutive pauses at the base station is given by

$$
T_{\text {total }}=\sum_{i} t_{i}+\sum_{i, j} t_{i j}
$$

where $t_{i}$ is the mean time that the UAV spends in the state $S_{i}$, for $i=0,1,2$.

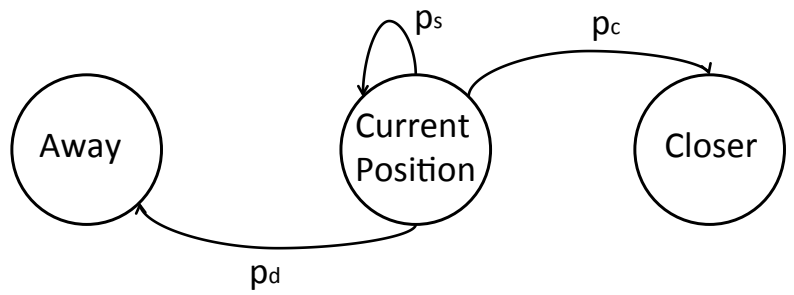

Fig. 4: The Markov chain in the $A_{1}, A_{3}$, and $A_{5}$ partitions. This model can capture the anomalies in the flight of the UAV when it is moving from a sensor to another or the base station.

Since the stationary probability shows the percentage of the time spent in the state $S_{i}$, the stationary distribution is given by

$$
\pi_{i}=\frac{t_{i}}{T_{\text {total }}}
$$

The UAV adjusts its trajectory depending on the location of the sensor $i$. The trajectory is determined such that the UAV collects the data from the sensor $i$ efficiently in terms of minimum flying time and maximum throughput. The UAV flies in the communication range of the sensor $i$ in height $h$ from it. The sensor $i$ starts transmitting $\lambda$ packets/slot when the UAV enters the communication range, so the number of packets that it transmits to the UAV is given by

$$
k=\lambda \cdot t_{i}
$$




\section{Numerical RESUlTS AND Discussions}

In this section, we provide numerical results to evaluate the success probability vs. the percentage of the distance that the UAV is moving in coverage of the sensor $i$. This distance is also the communication range of the sensor $i$. Table I and Table II show the distance from the sensor $i$, where the success probability is equal to $\delta=0.4$ and $\delta=0.7$ respectively.

Assuming that the UAV maintains the same height during its flight, we calculate the equations for each case presented in Table I. The equations for the cases where distance $=$ $173.9,31.1,138.3,26.7 \mathrm{~m}$ are $y=-x+176.5, y=-x+24.9$, $y=-x+73.9$ and $y=-x+21.5$ respectively. The equations for distance $=137.4,26.6,109.2,22.8$ in Table II are $y=-x+120.0, y=-x+20.3, y=-x+67.6$ and $y=-x+15.4$ respectively.

Fig. 5 shows the success probability vs. the percentage of the distance that the UAV has crossed for $\delta=0.4$ and for different values of $\gamma$ and $\alpha$. We can see that the success probability has the minimum value when the UAV is at the entry and exit point of the communication range. It has the maximum value when the UAV has the minimum distance from the sensor $i$ at $50 \%$ of the distance of the entry and exit point.

Fig. 6 shows the success probability vs. the percentage of the distance that the UAV has crossed for $\delta=0.7$ and we also examine it for different values of $\gamma$ and $\alpha$. We can extract the same observations for $\delta=0.7$ as we had for $\delta=0.4$. The success probability takes the minimum value at the entry and exit points. As the UAV travels from the entry point to the exit point, the success probability increases up to the middle point of this distance. After this point, it decreases and it reaches the minimum value as the UAV exits the communication range of the sensor $i$.

TABLE I: Parameters, distance and entry and exit points where success probability is equal to $\delta=0.4$

\begin{tabular}{c|c|c}
\hline Parameters & Distance [m] & Entry, Exit Point \\
\hline$\gamma=0.4, \alpha=4$ & 173.9 & $A(40.3,136.2,100.4), B(136.2,40.3,100.4)$ \\
$\gamma=0.4, \alpha=6$ & 31.1 & $A(5.1,19.8,23.4), B(19.8,5.1,23.4)$ \\
$\gamma=1, \alpha=4$ & 138.3 & $A(10.1,63.8,122.3), B(63.8,10.1,122.3)$ \\
$\gamma=1, \alpha=6$ & 26.7 & $A(6.7,14.8,21.2), B(14.8,6.7,21.2)$ \\
\hline
\end{tabular}

TABLE II: Parameters, distance and entry and exit points where success probability is equal to $\delta=0.7$

\begin{tabular}{c|c|c}
\hline Parameters & Distance $[\mathrm{m}]$ & Entry, Exit Point \\
\hline$\gamma=0.4, \alpha=4$ & 137.4 & $A(48.2,71.8,106.8), B(71.8,48.2,106.8)$ \\
$\gamma=0.4, \alpha=6$ & 26.6 & $A(3.2,17.1,20.1), B(17.1,3.2,20.1)$ \\
$\gamma=1, \alpha=4$ & 109.2 & $A(7.2,60.4,90.7), B(60.4,7.2,90.7)$ \\
$\gamma=1, \alpha=6$ & 22.8 & $A(2.1,13.3,18.4), B(13.3,2.1,18.4)$ \\
\hline
\end{tabular}

\section{Conclusion}

In this work we studied the performance of a UAV-assisted sensor network. We introduced a Markov chain to model the movement of the UAV where it visits at least one sensor to

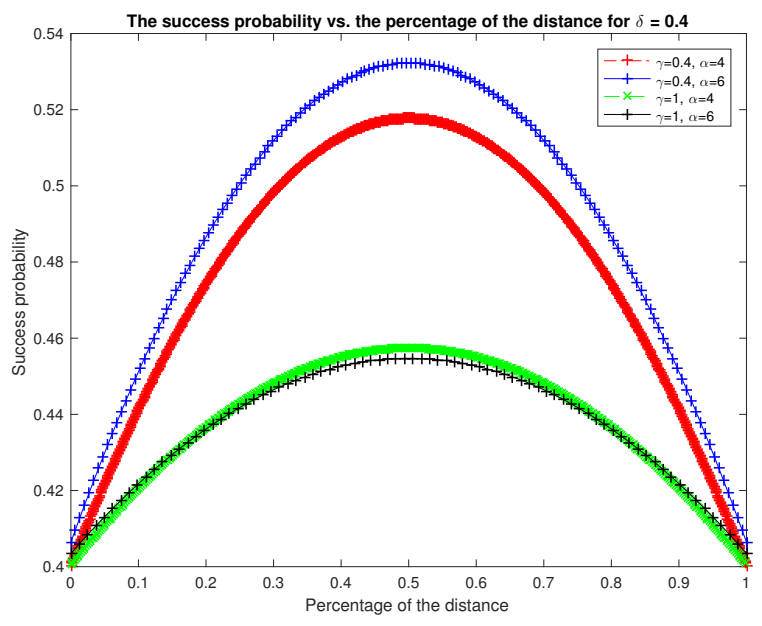

Fig. 5: The success probability vs. the percentage of the distance for $\delta=0.4$

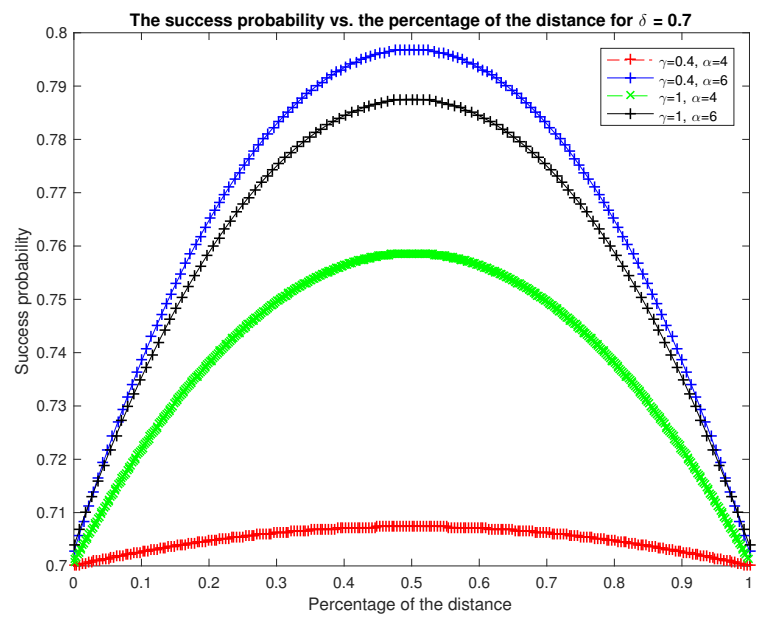

Fig. 6: The success probability vs. the percentage of the distance for $\delta=0.7$

collect data, communicates with it and travels back to a base station to transmit the data. We also proposed a second Markov chain model to model the deviation of the UAV's movement. We finally showed that the success probability that the UAV receives a packet is higher when the UAV reaches the 50\% of the flying distance. A future extension of our work will include energy harvesting, where the UAV can charge itself by renewable energy sources. Furthermore, the delay analysis on data collection will be performed.

\section{ACKNOWLEDGMENT}

This research work was funded in part by CUGS (the National Graduate School in Computer Science, Sweden and by the EU Project H2020 DECADE Nr. 645705. 


\section{REFERENCES}

[1] L. Gupta, R. Jain, and G. Vaszkun, "Survey of important issues in uav communication networks," IEEE Communications Surveys Tutorials, vol. 18, no. 2, pp. 1123-1152, Secondquarter 2016.

[2] Y. Zeng, R. Zhang, and T. J. Lim, "Wireless communications with unmanned aerial vehicles: opportunities and challenges," IEEE Communications Magazine, vol. 54, no. 5, pp. 36-42, May 2016.

[3] Z. M. Fadlullah, D. Takaishi, H. Nishiyama, N. Kato, and R. Miura, "A dynamic trajectory control algorithm for improving the communication throughput and delay in uav-aided networks," IEEE Network, vol. 30, no. 1, pp. 100-105, January 2016.

[4] E. Yanmaz, "Connectivity versus area coverage in unmanned aerial vehicle networks," in 2012 IEEE International Conference on Communications (ICC), June 2012, pp. 719-723.

[5] C. Dixon and E. W. Frew, "Optimizing cascaded chains of unmanned aircraft acting as communication relays," IEEE Journal on Selected Areas in Communications, vol. 30, no. 5, pp. 883-898, June 2012.

[6] D. T. Ho, E. I. Gr?tli, S. Shimamoto, and T. A. Johansen, "Optimal relay path selection and cooperative communication protocol for a swarm of uavs," in 2012 IEEE Globecom Workshops, Dec 2012, pp. 1585-1590.

[7] X. Li, D. Guo, H. Yin, and G. Wei, "Drone-assisted public safety wireless broadband network," in Wireless Communications and Networking Conference Workshops (WCNCW), 2015 IEEE, March 2015, pp. 323328.

[8] P. M. Olsson, J. Kvarnstrm, P. Doherty, O. Burdakov, and K. Holmberg, "Generating uav communication networks for monitoring and surveillance," in Control Automation Robotics Vision (ICARCV), 2010 11th International Conference on, Dec 2010, pp. 1070-1077.

[9] I. Jawhar, N. Mohamed, and J. Al-Jaroodi, "Uav-based data communication in wireless sensor networks: Models and strategies," pp. 687-694, June 2015.

[10] M. Horiuchi, H. Nishiyama, N. Kato, F. Ono, and R. Miura, "Throughput maximization for long-distance real-time data transmission over multiple uavs," in 2016 IEEE International Conference on Communications (ICC), May 2016, pp. 1-6.

[11] F. Ono, H. Ochiai, and R. Miura, "A wireless relay network based on unmanned aircraft system with rate optimization," IEEE Transactions on Wireless Communications, vol. PP, no. 99, pp. 1-1, 2016.

[12] A. Giorgetti, M. Lucchi, M. Chiani, and M. Z. Win, "Throughput per pass for data aggregation from a wireless sensor network via a uav," IEEE Transactions on Aerospace and Electronic Systems, vol. 47, no. 4, pp. 2610-2626, OCTOBER 2011.

[13] M. Mozaffari, W. Saad, M. Bennis, and M. Debbah, "Efficient deployment of multiple unmanned aerial vehicles for optimal wireless coverage," IEEE Communications Letters, vol. 20, no. 8, pp. 1647-1650, Aug 2016.

[14] M. Chen, M. Mozaffari, W. Saad, C. Yin, M. Debbah, , and C. S. Hong, "Caching in the sky: Proactive deployment of cache-enabled unmanned aerial vehicles for optimized quality-of-experience," to appear.

[15] K. Li, W. Ni, X. Wang, R. P. Liu, S. S. Kanhere, and S. Jha, "Energy-efficient cooperative relaying for unmanned aerial vehicles," IEEE Transactions on Mobile Computing, vol. 15, no. 6, pp. 1377-1386, June 2016.

[16] _ _ "Epla: Energy-balancing packets scheduling for airborne relaying networks," in 2015 IEEE International Conference on Communications (ICC), June 2015, pp. 6246-6251.

[17] J. Kwon and S. Hailes, "Scheduling uavs to bridge communications in delay-tolerant networks using real-time scheduling analysis techniques," in System Integration (SII), 2014 IEEE/SICE International Symposium on, Dec 2014, pp. 363-369.

[18] N. D. Powel and K. A. Morgansen, "Multiserver queueing for supervisory control of autonomous vehicles," in 2012 American Control Conference (ACC), June 2012, pp. 3179-3185.

[19] E. Yanmaz, R. Kuschnig, and C. Bettstetter, "Achieving air-ground communications in 802.11 networks with three-dimensional aerial mobility," in INFOCOM, 2013 Proceedings IEEE, April 2013, pp. 120-124.

[20] S. Jeong, O. Simeone, and J. Kang, "Mobile edge computing via a uavmounted cloudlet: Optimal bit allocation and path planning," to appear.

[21] E. Yanmaz, C. Costanzo, C. Bettstetter, and W. Elmenreich, "A discrete stochastic process for coverage analysis of autonomous uav networks," in 2010 IEEE Globecom Workshops, Dec 2010, pp. 1777-1782.
[22] "The future of uavs (drones) in data collection," http://blog.junipersys.com/future-of-uavs-drones-in-data-collection/, accessed: 2017-03-12.

[23] J. Lloret, J. Tomas, A. Canovas, and L. Parra, "An integrated iot architecture for smart metering," IEEE Communications Magazine, vol. 54, no. 12 , pp. 50-57, December 2016. 